\title{
On Jacobsthal and Jacobsthal-Lucas Circulant Type Matrices
}

\author{
Yanpeng Gong, Zhaolin Jiang, and Yun Gao \\ Department of Mathematics, Linyi University, Linyi, Shandong 276005, China \\ Correspondence should be addressed to Zhaolin Jiang; jzh1208@sina.com
}

Received 27 June 2014; Revised 19 October 2014; Accepted 19 October 2014

Academic Editor: Shurong Sun

Copyright (C) 2015 Yanpeng Gong et al. This is an open access article distributed under the Creative Commons Attribution License, which permits unrestricted use, distribution, and reproduction in any medium, provided the original work is properly cited.

\begin{abstract}
Circulant type matrices have become an important tool in solving fractional order differential equations. In this paper, we consider the circulant and left circulant and $g$-circulant matrices with the Jacobsthal and Jacobsthal-Lucas numbers. First, we discuss the invertibility of the circulant matrix and present the determinant and the inverse matrix. Furthermore, the invertibility of the left circulant and $g$-circulant matrices is also discussed. We obtain the determinants and the inverse matrices of the left circulant and $g$-circulant matrices by utilizing the relation between left circulant, $g$-circulant matrices, and circulant matrix, respectively.
\end{abstract}

\section{Introduction}

Circulant type matrices possess interesting properties, which have been exploited to obtain the transient solution in a closed form for fractional order differential equations. In [1], $\mathrm{Qu}$ et al. derived the discretized linear system from fractional advection-diffusion equations (FADEs) with constant coefficients and introduced the background of circulant and skew-circulant splitting (CSCS) iteration for solving the discretized linear system. In [2], some Routh-Hurwitz stability conditions are generalized to the fractional order case. Ahmed et al. considered the 1-system CML (10) in [2]. They selected a circulant matrix, which reads a tridiagonal matrix. Ahmed et al. used coupled map lattices (CML) as an alternative approach to include spatial effects in fractional order systems (FOS). Consider the 1-system CML (10) in [3]. They claimed that the system is stable if all the eigenvalues of the circulant matrix satisfy (2) in [3]. Lei and Sun [4] proposed the preconditioned CGNR (PCGNR) method with a circulant preconditioner to solve such Toeplitz-like systems. Kloeden et al. adopted the multilevel Monte Carlo method introduced by M. Giles to SDEs with additive fractional noise. They adopted the simplest approximation schemes for (1) in [5] with the Euler method, which reads (5) in [5]. They exploited that the covariance matrix of the increments can be embedded in a circulant matrix. The total loops can be done by fast Fourier transformation, which leads to a total computational cost of $O(m \log m)$, where $m$ is the order of the circulant matrix.

Jacobsthal and Jacobsthal-Lucas circulant type matrices are circulant type matrices with Jacobsthal and JacobsthalLucas numbers. Circulant type matrices include the circulant and left circulant and $g$-circulant matrices. These matrices have important applications in various disciplines including image processing, communications, signal processing, encoding, solving Toeplitz matrix problems, preconditioner, and solving least squares problems. They have been put on the firm basis with the work of Davis [6] and Jiang and Zhou [7].

The $g$-circulant matrices play an important role in various applications as well; for details please refer to $[8,9]$ and the references therein. Ngondiep et al. showed the singular values of $g$-circulants in [10]. There are discussions about the convergence in probability and in distribution of the spectral norm of $g$-circulant matrices in [11].

For $n>0$, the Jacobsthal number $\left\{J_{n}\right\}$ is defined by $J_{n+1}=$ $J_{n}+2 J_{n-1}$, where $J_{0}=0$ and $J_{1}=1$. If we generalize $J_{n+1}$ to $j_{n+1}=j_{n}+2 j_{n-1}$ and let $j_{0}=2, j_{1}=1$, then we obtain Jacobsthal-Lucas number $\left\{j_{n}\right\}$. Let $\alpha$ and $\beta$ be the roots of the characteristic equation $x^{2}-x-2=0$; then the Binet formulas of the sequences $\left\{J_{n}\right\}$ and $\left\{j_{n}\right\}$ have the form $[12,13]$

$$
J_{n}=\frac{\alpha^{n}-\beta^{n}}{\alpha-\beta}, \quad j_{n}=\alpha^{n}+\beta^{n}
$$


Besides, some scholars have given various algorithms for the determinants and inverses of nonsingular circulant matrices in [6]. It is worth pointing out that the computational complexity of these algorithms is amazing with the order of matrix increasing. However, some authors gave the explicit determinant and inverse of the circulant and skewcirculant matrix involving Fibonacci and Lucas numbers. For example, authors [14] studied the nonsingularity of the skewcirculant type matrices and presented explicit determinants and inverse matrices of these special matrices. Furthermore, four kinds of norms and bounds for the spread of these matrices are given separately. In [15], the nonsingularity of circulant type matrices with the sum and product of Fibonacci and Lucas numbers is discussed. And the exact determinants and inverses of these matrices are given. In [16], Jiang and Hong presented exact determinants of some special circulant matrices involving four kinds of famous numbers. Jaiswal evaluated some determinants of circulant matrix whose elements are the generalized Fibonacci numbers in [17]. Lind presented the determinants of circulant and skew-circulant matrix involving Fibonacci numbers in [18]. Dazheng gave the determinant of the Fibonacci-Lucas quasicyclic matrices in [19]. Shen et al. considered circulant matrices with Fibonacci and Lucas numbers and presented their explicit determinants and inverses by constructing the transformation matrices in [20].

The purpose of this paper is to obtain the better results for the determinants and inverses of circulant type matrices by some properties of Jacobsthal and Jacobsthal-Lucas numbers.

Definition 1 (see [21]). A right circulant matrix (or simply, circulant matrix) is written as

$$
\operatorname{Circ}\left(a_{1}, a_{2}, \ldots, a_{n}\right)=\left[\begin{array}{cccc}
a_{1} & a_{2} & \cdots & a_{n} \\
a_{n} & a_{1} & \cdots & a_{n-1} \\
\vdots & \vdots & & \vdots \\
a_{2} & a_{3} & \cdots & a_{1}
\end{array}\right] .
$$

Definition 2 (see [21]). A left circulant matrix is written as

$$
\operatorname{LCirc}\left(a_{1}, a_{2}, \ldots, a_{n}\right)=\left[\begin{array}{cccc}
a_{1} & a_{2} & \cdots & a_{n} \\
a_{2} & a_{3} & \cdots & a_{1} \\
\vdots & \vdots & & \vdots \\
a_{n} & a_{1} & \cdots & a_{n-1}
\end{array}\right] .
$$

Definition 3 (see [22]). A $g$-circulant matrix is an $n \times n$ complex matrix with the following form:

$$
A_{g, n}=\left(\begin{array}{cccc}
a_{1} & a_{2} & \cdots & a_{n} \\
a_{n-g+1} & a_{n-g+2} & \cdots & a_{n-g} \\
a_{n-2 g+1} & a_{n-2 g+2} & \cdots & a_{n-2 g} \\
\vdots & \vdots & \ddots & \vdots \\
a_{g+1} & a_{g+2} & \cdots & a_{g}
\end{array}\right),
$$

where $g$ is a nonnegative integer and each of the subscripts is understood to be reduced modulo $n$.

The first row of $A_{g, n}$ is $\left(a_{1}, a_{2}, \ldots, a_{n}\right)$; its $(j+1)$ th row is obtained by giving its $j$ th row a right circular shift by $g$ positions (equivalently, $g$ mod $n$ positions). Note that $g=1$ or $g=n+1$ yields the standard circulant matrix. If $g=n-1$, then we obtain the left circulant matrix.

\section{Circulant Matrix with the Jacobsthal Numbers}

In this section, let $A_{n}=\operatorname{Circ}\left(J_{1}, J_{2}, \ldots, J_{n}\right)$ be a circulant matrix. Firstly, we give a determinant formula for the matrix $A_{n}$. Afterwards, we prove that $A_{n}$ is an invertible matrix for $n>2$, and then we find the inverse of the matrix $A_{n}$.

Theorem 4. Let $A_{n}=\operatorname{Circ}\left(J_{1}, J_{2}, \ldots, J_{n}\right)$ be a circulant matrix; then we have

$$
\begin{aligned}
\operatorname{det} A_{n}= & \left(1-J_{n+1}\right)^{n-1} \\
& +\left(2 J_{n}\right)^{n-2} \sum_{k=1}^{n-1}\left(2 J_{k}\right)\left(\frac{1-J_{n+1}}{2 J_{n}}\right)^{k-1},
\end{aligned}
$$

where $J_{n}$ is the nth Jacobsthal number.

Proof. Obviously, when $n=1$, $\operatorname{det} A_{1}=1$ satisfies the formula (5). In the case $n>1$, let

$$
\begin{aligned}
& \Gamma=\left(\begin{array}{cccccccc}
1 & & & & & & \\
-1 & & & & & & 1 \\
-2 & & & & & 1 & -1 \\
0 & & 0 & & 1 & -1 & -2 \\
\vdots & & & & \ddots & \ddots & . \\
0 & & 1 & \ddots & \ddots & \\
0 & & 1 & -1 & \ddots & & 0 & \\
0 & 1 & -1 & -2 & & & &
\end{array}\right) \text {, } \\
& \Pi_{1}=\left(\begin{array}{cccccc}
1 & 0 & 0 & \cdots & 0 & 0 \\
0 & \left(\frac{2 J_{n}}{J_{1}-J_{n+1}}\right)^{n-2} & 0 & \cdots & 0 & 1 \\
0 & \left(\frac{2 J_{n}}{J_{1}-J_{n+1}}\right)^{n-3} & 0 & \cdots & 1 & 0 \\
\vdots & \vdots & \vdots & \ddots & \vdots & \vdots \\
0 & \frac{2 J_{n}}{J_{1}-J_{n+1}} & 1 & \cdots & 0 & 0 \\
0 & 1 & 0 & \cdots & 0 & 0
\end{array}\right)
\end{aligned}
$$

be two $n \times n$ matrices; then the product $\Gamma A_{n} \Pi_{1}$ has the form

$$
\left(\begin{array}{cccccc}
J_{1} & \delta_{n}^{\prime} & J_{n-1} & \cdots & J_{3} & J_{2} \\
0 & \delta_{n} & 2 J_{n-2} & \cdots & 2 J_{2} & 2 J_{1} \\
0 & 0 & J_{1}-J_{n+1} & & & 0 \\
0 & 0 & -2 J_{n} & \ddots & & 0 \\
\vdots & \vdots & & \ddots & \ddots & \\
0 & 0 & & & & \\
0 & 0 & 0 & & -2 J_{n} & J_{1}-J_{n+1}
\end{array}\right),
$$


where

$$
\begin{gathered}
\delta_{n}=J_{1}-J_{n}+\sum_{k=1}^{n-2} 2 J_{k}\left(\frac{2 J_{n}}{J_{1}-J_{n+1}}\right)^{n-(k+1)}, \\
\delta_{n}^{\prime}=\sum_{k=1}^{n-1} J_{k+1}\left(\frac{2 J_{n}}{J_{1}-J_{n+1}}\right)^{n-(k+1)} .
\end{gathered}
$$

So we obtain

$\operatorname{det} \Gamma \operatorname{det} A_{n} \operatorname{det} \Pi_{1}$

$$
\begin{aligned}
= & J_{1} \times\left[J_{1}-J_{n}+\sum_{k=1}^{n-2} 2 J_{k}\left(\frac{2 J_{n}}{J_{1}-J_{n+1}}\right)^{n-(k+1)}\right] \\
& \times\left(J_{1}-J_{n+1}\right)^{n-2} \\
= & J_{1} \times\left[J_{1}-J_{n+1}+\sum_{k=1}^{n-1} 2 J_{k}\left(\frac{2 J_{n}}{J_{1}-J_{n+1}}\right)^{n-(k+1)}\right] \\
& \times\left(J_{1}-J_{n+1}\right)^{n-2} \\
= & \left(1-J_{n+1}\right)^{n-1}+\left(2 J_{n}\right)^{n-2} \sum_{k=1}^{n-1} 2 J_{k}\left(\frac{1-J_{n+1}}{2 J_{n}}\right)^{k-1},
\end{aligned}
$$

while

$$
\operatorname{det} \Gamma=\operatorname{det} \Pi_{1}=(-1)^{((n-1)(n-2)) / 2} \text {, }
$$

and we have

$$
\operatorname{det} A_{n}=\left(1-J_{n+1}\right)^{n-1}+\left(2 J_{n}\right)^{n-2} \sum_{k=1}^{n-1} 2 J_{k}\left(\frac{1-J_{n+1}}{2 J_{n}}\right)^{k-1}
$$

The proof is completed.

Theorem 5. Let $A_{n}=\operatorname{Circ}\left(J_{1}, J_{2}, \ldots, J_{n}\right)$ be a circulant matrix. If $n>2$, then $A_{n}$ is an invertible matrix.

Proof. When $n=3$ in Theorem 4, then we have $\operatorname{det} A_{3}=$ $20 \neq 0$; hence $A_{3}$ is invertible. In the case $n>3$, since $J_{n}=$ $\left(\alpha^{n}-\beta^{n}\right) /(\alpha-\beta)$, where $\alpha+\beta=1, \alpha \beta=-2$, we have

$$
\begin{aligned}
f\left(\omega^{k}\right) & =\sum_{i=1}^{n} J_{i}\left(\omega^{k}\right)^{i-1} \\
& =\frac{1}{\alpha-\beta} \sum_{i=1}^{n}\left(\alpha^{i}-\beta^{i}\right)\left(\omega^{k}\right)^{i-1}
\end{aligned}
$$

$$
\begin{gathered}
=\frac{1}{\alpha-\beta}\left[\frac{\alpha\left(1-\alpha^{n}\right)}{1-\alpha \omega^{k}}-\frac{\beta\left(1-\beta^{n}\right)}{1-\beta \omega^{k}}\right] \\
=\frac{1}{\alpha-\beta}\left[\frac{(\alpha-\beta)-\left(\alpha^{n+1}-\beta^{n+1}\right)}{1-(\alpha+\beta) \omega^{k}+\alpha \beta \omega^{2 k}}\right. \\
\left.\quad+\frac{\alpha \beta\left(\alpha^{n}-\beta^{n}\right) \omega^{k}}{1-(\alpha+\beta) \omega^{k}+\alpha \beta \omega^{2 k}}\right] \\
=\frac{1-J_{n+1}-2 J_{n} \omega^{k}}{1-\omega^{k}-2 \omega^{2 k}} \quad(k=1,2, \ldots, n-1) .
\end{gathered}
$$

If there exists $\omega^{l}(l=1,2, \ldots, n-1)$ such that $f\left(\omega^{l}\right)=0$, then we obtain $1-J_{n+1}-2 J_{n} \omega^{l}=0$ for $1-\omega^{l}-2 \omega^{2 l} \neq 0$; thus, $\omega^{l}=\left(1-J_{n+1}\right) / 2 J_{n}$ is a real number. While

$$
\omega^{l}=\exp \left(\frac{2 l \pi i}{n}\right)=\cos \frac{2 l \pi}{n}+i \sin \frac{2 l \pi}{n},
$$

$\sin (2 l \pi / n)=0$, so we have $\omega^{l}=-1$ for $0<2 l \pi / n<2 \pi$. But $x=-1$ is not the root of the equation $1-J_{n+1}-2 J_{n} x=$ $0 \quad(n>3)$. We obtain $f\left(\omega^{k}\right) \neq 0$ for any $\omega^{k}(k=1,2, \ldots, n-$ $1)$, while $f(1)=\sum_{i=1}^{n} J_{i}=-(1 / 2)\left(1-J_{n+1}-2 J_{n}\right) \neq 0$. By Lemma 4 in [15], the proof is completed.

Lemma 6. Let the matrix $\mathscr{G}=\left[g_{i j}\right]_{i, j=1}^{n-2}$ be of the form

$$
g_{i j}= \begin{cases}J_{1}-J_{n+1}, & i=j, \\ -2 J_{n}, & i=j+1, \\ 0, & \text { otherwise }\end{cases}
$$

and then the inverse $\mathscr{G}^{-1}=\left[g_{i j}^{\prime}\right]_{i, j=1}^{n-2}$ is equal to

$$
g_{i j}^{\prime}= \begin{cases}\frac{\left(2 J_{n}\right)^{i-j}}{\left(J_{1}-J_{n+1}\right)^{i-j+1}}, & i \geq j, \\ 0, & i<j .\end{cases}
$$

Proof. Let $c_{i j}=\sum_{k=1}^{n-2} g_{i k} g_{k j}^{\prime}$. Obviously, $c_{i j}=0$ for $i<j$. In the case $i=j$, we obtain

$$
c_{i i}=g_{i i} g_{i i}^{\prime}=\left(J_{1}-J_{n+1}\right) \cdot \frac{1}{J_{1}-J_{n+1}}=1 .
$$

For $i \geq j+1$, we obtain

$$
\begin{aligned}
c_{i j}= & \sum_{k=1}^{n-2} g_{i k} g_{k j}^{\prime}=g_{i, i-1} g_{i-1, j}^{\prime}+g_{i i} g_{i j}^{\prime} \\
= & \left(-2 J_{n}\right) \cdot \frac{\left(2 J_{n}\right)^{i-j-1}}{\left(J_{1}-J_{n+1}\right)^{i-j}} \\
& +\left(J_{1}-J_{n+1}\right) \cdot \frac{\left(2 J_{n}\right)^{i-j}}{\left(J_{1}-J_{n+1}\right)^{i-j+1}}=0 .
\end{aligned}
$$

Hence, we verify $\mathscr{G} \mathscr{G}^{-1}=I_{n-2}$, where $I_{n-2}$ is $(n-2) \times(n-2)$ identity matrix. Similarly, we can verify $\mathscr{G}^{-1} \mathscr{G}=I_{n-2}$. Thus, the proof is completed. 
Theorem 7. Let $A_{n}=\operatorname{Circ}\left(J_{1}, J_{2}, \ldots, J_{n}\right)(n>2)$ be a circulant matrix; then one has

$$
A_{n}^{-1}=\frac{1}{\delta_{n}} \operatorname{Circ}\left(x_{1}^{\prime}, x_{2}^{\prime}, \ldots, x_{n}^{\prime}\right)
$$

where

$$
\begin{aligned}
& x_{1}^{\prime}=1+2 \sum_{i=1}^{n-2} \frac{J_{n-i}\left(2 J_{n}\right)^{i-1}}{\left(J_{1}-J_{n+1}\right)^{i}}, \\
& x_{2}^{\prime}=-1+4 \sum_{i=1}^{n-2} \frac{J_{n-1-i}\left(2 J_{n}\right)^{i-1}}{\left(J_{1}-J_{n+1}\right)^{i}}, \\
& x_{k}^{\prime}=-\frac{2\left(2 J_{n}\right)^{k-3}}{\left(J_{1}-J_{n+1}\right)^{k-2}}, \quad k=3,4, \ldots, n, \\
& \delta_{n}=J_{1}-J_{n}+\sum_{k=1}^{n-2} 2 J_{k}\left(\frac{2 J_{n}}{J_{1}-J_{n+1}}\right)^{n-(k+1)} .
\end{aligned}
$$

Proof. Let

$$
\Pi_{2}=\left(\begin{array}{ccccc}
1 & -\delta_{n}^{\prime} & 2 \frac{\delta_{n}^{\prime}}{\delta_{n}} J_{n-2}-J_{n-1} & \cdots & 2 \frac{\delta_{n}^{\prime}}{\delta_{n}} J_{1}-J_{2} \\
0 & 1 & -2 \frac{J_{n-2}}{\delta_{n}} & \cdots & -2 \frac{J_{1}}{\delta_{n}} \\
0 & 0 & 1 & \cdots & 0 \\
0 & 0 & 0 & \cdots & 0 \\
\vdots & \vdots & \vdots & \ddots & \vdots \\
0 & 0 & 0 & \cdots & 1
\end{array}\right),
$$

where

$$
\begin{gathered}
\delta_{n}=J_{1}-J_{n}+\sum_{k=1}^{n-2} 2 J_{k}\left(\frac{2 J_{n}}{J_{1}-J_{n+1}}\right)^{n-(k+1)}, \\
\delta_{n}^{\prime}=\sum_{k=1}^{n-1} J_{k+1}\left(\frac{2 J_{n}}{J_{1}-J_{n+1}}\right)^{n-(k+1)} .
\end{gathered}
$$

Then we have

$$
\Gamma A_{n} \Pi_{1} \Pi_{2}=\mathscr{D}_{1} \oplus \mathscr{G}
$$

where $\mathscr{D}_{1}=\operatorname{diag}\left(J_{1}, \delta_{n}\right)$ is a diagonal matrix, and $\mathscr{D}_{1} \oplus \mathscr{G}$ is the direct sum of $\mathscr{D}_{1}$ and $\mathscr{G}$. If we denote $\Pi=\Pi_{1} \Pi_{2}$, then we obtain

$$
A_{n}^{-1}=\Pi\left(\mathscr{D}_{1}^{-1} \oplus \mathscr{G}^{-1}\right) \Gamma
$$

since the last row entries of the matrix $\Pi$ are $0,1,-2\left(J_{n-2} /\right.$ $\left.\delta_{n}\right),-2\left(J_{n-3} / \delta_{n}\right), \ldots,-2\left(J_{2} / \delta_{n}\right),-2\left(J_{1} / \delta_{n}\right)$. By Lemma 6 , if we let $A_{n}^{-1}=\operatorname{Circ}\left(x_{1}, x_{2}, \ldots, x_{n}\right)$, then its last row entries are given by the following equations:

$$
x_{2}=-\frac{1}{\delta_{n}}+\frac{4}{\delta_{n}} \sum_{i=1}^{n-2} \frac{J_{n-1-i}\left(2 J_{n}\right)^{i-1}}{\left(J_{1}-J_{n+1}\right)^{i}} \text {, }
$$

$$
\begin{aligned}
x_{3}= & -\frac{2 J_{1}}{\delta_{n}\left(J_{1}-J_{n+1}\right)}, \\
x_{4}= & -\frac{2}{\delta_{n}} \sum_{i=1}^{2} \frac{J_{3-i}\left(2 J_{n}\right)^{i-1}}{\left(J_{1}-J_{n+1}\right)^{i}}+\frac{2 J_{1}}{\delta_{n}\left(J_{1}-J_{n+1}\right)}, \\
x_{5}= & -\frac{2}{\delta_{n}} \sum_{i=1}^{3} \frac{J_{4-i}\left(2 J_{n}\right)^{i-1}}{\left(J_{1}-J_{n+1}\right)^{i}}+\frac{2}{\delta_{n}} \sum_{i=1}^{2} \frac{J_{3-i}\left(2 J_{n}\right)^{i-1}}{\left(J_{1}-J_{n+1}\right)^{i}} \\
& +\frac{4 J_{1}}{\delta_{n}\left(J_{1}-J_{n+1}\right)},
\end{aligned}
$$

$$
\vdots
$$$$
x_{n}=-\frac{2}{\delta_{n}} \sum_{i=1}^{n-2} \frac{J_{n-1-i}\left(2 J_{n}\right)^{i-1}}{\left(J_{1}-J_{n+1}\right)^{i}}+\frac{2}{\delta_{n}} \sum_{i=1}^{n-3} \frac{J_{n-2-i}\left(2 J_{n}\right)^{i-1}}{\left(J_{1}-J_{n+1}\right)^{i}}
$$$$
+\frac{4}{\delta_{n}} \sum_{i=1}^{n-4} \frac{J_{n-3-i}\left(2 J_{n}\right)^{i-1}}{\left(J_{1}-J_{n+1}\right)^{i}}
$$$$
x_{1}=\frac{1}{\delta_{n}}+\frac{2}{\delta_{n}} \sum_{i=1}^{n-2} \frac{J_{n-1-i}\left(2 J_{n}\right)^{i-1}}{\left(J_{1}-J_{n+1}\right)^{i}}+\frac{4}{\delta_{n}} \sum_{i=1}^{n-3} \frac{J_{n-2-i}\left(2 J_{n}\right)^{i-1}}{\left(J_{1}-J_{n+1}\right)^{i}} .
$$

Let $C_{n}^{(k)}=\sum_{i=1}^{k}\left(J_{k+1-i}\left(2 J_{n}\right)^{i-1} /\left(J_{1}-J_{n+1}\right)^{i}\right),(j=1,2, \ldots, n-$ 2); then we have

$$
\begin{aligned}
& C_{n}^{(2)}-C_{n}^{(1)} \\
& =\sum_{i=1}^{2} \frac{J_{3-i}\left(2 J_{n}\right)^{i-1}}{\left(J_{1}-J_{n+1}\right)^{i}}-\frac{J_{1}}{J_{1}-J_{n+1}}=\frac{2 J_{n}}{\left(J_{1}-J_{n+1}\right)^{2}} \\
& C_{n}^{(n-2)}+2 C_{n}^{(n-3)} \\
& =\sum_{i=1}^{n-2} \frac{J_{n-1-i}\left(2 J_{n}\right)^{i-1}}{\left(J_{1}-J_{n+1}\right)^{i}}+2 \sum_{i=1}^{n-3} \frac{J_{n-2-i}\left(2 J_{n}\right)^{i-1}}{\left(J_{1}-J_{n+1}\right)^{i}} \\
& =\frac{J_{1}\left(2 J_{n}\right)^{n-3}}{\left(J_{1}-J_{n+1}\right)^{n-2}}+\sum_{i=1}^{n-3} \frac{J_{n-i}\left(2 J_{n}\right)^{i-1}}{\left(J_{1}-J_{n+1}\right)^{i}} \\
& \quad=\sum_{i=1}^{n-2} \frac{J_{n-i}\left(2 J_{n}\right)^{i-1}}{\left(J_{1}-J_{n+1}\right)^{i}}, \\
& C_{n}^{(k+2)}-C_{n}^{(k+1)}-2 C_{n}^{(k)} \\
& =\sum_{i=1}^{k+2} \frac{J_{k+3-i}\left(2 J_{n}\right)^{i-1}}{\left(J_{1}-J_{n+1}\right)^{i}}
\end{aligned}
$$




$$
\begin{aligned}
& -\sum_{i=1}^{k+1} \frac{J_{k+2-i}\left(2 J_{n}\right)^{i-1}}{\left(J_{1}-J_{n+1}\right)^{i}}-2 \sum_{i=1}^{k} \frac{J_{k+1-i}\left(2 J_{n}\right)^{i-1}}{\left(J_{1}-J_{n+1}\right)^{i}} \\
= & \frac{J_{2}\left(2 J_{n}\right)^{k}}{\left(J_{1}-J_{n+1}\right)^{k+1}}+\frac{J_{1}\left(2 J_{n}\right)^{k+1}}{\left(J_{1}-J_{n+1}\right)^{k+2}} \\
& -\frac{J_{1}\left(2 J_{n}\right)^{k}}{\left(J_{1}-J_{n+1}\right)^{k+1}} \\
& +\sum_{i=1}^{k} \frac{\left(J_{k+3-i}-J_{k+2-i}-2 J_{k+1-i}\right)\left(2 J_{n}\right)^{i-1}}{\left(J_{1}-J_{n+1}\right)^{i}} \\
= & \frac{\left(2 J_{n}\right)^{k+1}}{\left(J_{1}-J_{n+1}\right)^{k+2}} \quad(k=1,2, \ldots, n-4) .
\end{aligned}
$$

We obtain

$$
\begin{aligned}
& x_{1}=\frac{1+2\left(C_{n}^{(n-2)}+2 C_{n}^{(n-3)}\right)}{\delta_{n}}, \\
& x_{2}=\frac{4 C_{n}^{(n-2)}-1}{\delta_{n}}, \\
& x_{3}=-\frac{2 C_{n}^{(1)}}{\delta_{n}}, \\
& x_{4}=-\frac{2\left(C_{n}^{(2)}-C_{n}^{(1)}\right)}{\delta_{n}}, \\
& x_{k}=-\frac{2\left(C_{n}^{(k-2)}-C_{n}^{(k-3)}-2 C_{n}^{(k-4)}\right)}{\delta_{n}}, \quad k=5,6, \ldots, n, \\
& A_{n}^{-1}=\frac{1}{\delta_{n}} \operatorname{Circ}\left(x_{1}^{\prime}, x_{2}^{\prime}, \ldots, x_{n}^{\prime}\right),
\end{aligned}
$$

where

$$
\begin{aligned}
& x_{1}^{\prime}=1+2 \sum_{i=1}^{n-2} \frac{J_{n-i}\left(2 J_{n}\right)^{i-1}}{\left(J_{1}-J_{n+1}\right)^{i}}, \\
& x_{2}^{\prime}=-1+4 \sum_{i=1}^{n-2} \frac{J_{n-1-i}\left(2 J_{n}\right)^{i-1}}{\left(J_{1}-J_{n+1}\right)^{i}}, \\
& x_{k}^{\prime}=-\frac{2\left(2 J_{n}\right)^{k-3}}{\left(J_{1}-J_{n+1}\right)^{k-2}}, \quad k=3,4, \ldots, n .
\end{aligned}
$$

The proof is completed.

\section{Circulant Matrix with the Jacobsthal-Lucas Numbers}

In this section, let $B_{n}=\operatorname{Circ}\left(j_{1}, j_{2}, \ldots, j_{n}\right)$ be a circulant matrix. Firstly, we give a determinant formula for the matrix
$B_{n}$. Then, we prove that $B_{n}$ is an invertible matrix for any positive integer $n$, and finally we find the inverse of the matrix $B_{n}$.

Theorem 8. Let $B_{n}=\operatorname{Circ}\left(j_{1}, j_{2}, \ldots, j_{n}\right)$ be a circulant matrix; then one has

$$
\begin{aligned}
\operatorname{det} B_{n}= & \left(1-j_{n+1}\right)^{n-1}+\left(2 j_{n}-4\right)^{n-2} \\
& \times \sum_{k=1}^{n-1}\left(j_{k+2}-5 j_{k+1}\right)\left(\frac{1-j_{n+1}}{2 j_{n}-4}\right)^{k-1},
\end{aligned}
$$

where $j_{n}$ is the nth Jacobsthal-Lucas number.

Proof. Obviously, when $n=1$, $\operatorname{det} B_{1}=1$ satisfies the formula (28). When $n>1$, let

$$
\Sigma=\left(\begin{array}{ccccccccc}
1 & & & & & & & \\
-5 & & & & & & 1 \\
-2 & & & & & 1 & -1 \\
0 & & 0 & & 1 & -1 & -2 \\
\vdots & & & & . & . & . & \\
0 & & & 1 & . & . & . & & \\
0 & & 1 & -1 & . & & 0 & \\
0 & 1 & -1 & -2 & & & &
\end{array}\right) \text {, }
$$

$$
\Omega_{1}=\left(\begin{array}{cccccc}
1 & 0 & 0 & \cdots & 0 & 0 \\
0 & \left(\frac{2 j_{n}-4}{j_{1}-j_{n+1}}\right)^{n-2} & 0 & \cdots & 0 & 1 \\
0 & \left(\frac{2 j_{n}-4}{j_{1}-j_{n+1}}\right)^{n-3} & 0 & \cdots & 1 & 0 \\
\vdots & \vdots & \vdots & \ddots & \vdots & \vdots \\
0 & \frac{2 j_{n}-4}{j_{1}-j_{n+1}} & 1 & \cdots & 0 & 0 \\
0 & 1 & 0 & \cdots & 0 & 0
\end{array}\right)
$$

be two $n \times n$ matrices; then the product $\Sigma B_{n} \Omega_{1}$ has the form

$$
\left(\begin{array}{cccccc}
j_{1} & \gamma_{n}^{\prime} & j_{n-1} & \cdots & j_{3} & j_{2} \\
0 & \gamma_{n} & j_{n}-5 j_{n-1} & \cdots & j_{4}-5 j_{3} & j_{3}-5 j_{2} \\
0 & 0 & j_{1}-j_{n+1} & & & \\
0 & 0 & 4-2 j_{n} & \ddots & & 0 \\
\vdots & \vdots & & \ddots & \ddots & \\
0 & 0 & & & 4-2 j_{n} & j_{1}-j_{n+1}
\end{array}\right),
$$

where

$$
\begin{gathered}
\gamma_{n}=j_{1}-5 j_{n}+\sum_{k=1}^{n-2}\left(j_{k+2}-5 j_{k+1}\right)\left(\frac{2 j_{n}-4}{j_{1}-j_{n+1}}\right)^{n-(k+1)}, \\
\gamma_{n}^{\prime}=\sum_{k=1}^{n-1} j_{k+1}\left(\frac{2 j_{n}-4}{j_{1}-j_{n+1}}\right)^{n-(k+1)} .
\end{gathered}
$$


We obtain

$$
\begin{aligned}
& \operatorname{det} \Sigma \operatorname{det} B_{n} \operatorname{det} \Omega_{1} \\
&=j_{1} \times\left[j_{1}-5 j_{n}+\sum_{k=1}^{n-2}\left(j_{k+2}-5 j_{k+1}\right)\right. \\
&\left.\times\left(\frac{2 j_{n}-4}{j_{1}-j_{n+1}}\right)^{n-(k+1)}\right] \times\left(j_{1}-j_{n+1}\right)^{n-2} \\
&=j_{1} \times\left[j_{1}-j_{n+1}+\sum_{k=1}^{n-1}\left(j_{k+2}-5 j_{k+1}\right)\right. \\
&\left.\times\left(\frac{2 j_{n}-4}{j_{1}-j_{n+1}}\right)^{n-(k+1)}\right] \times\left(j_{1}-j_{n+1}\right)^{n-2} \\
&=\left(1-j_{n+1}\right)^{n-1}+\left(2 j_{n}-4\right)^{n-2} \sum_{k=1}^{n-1}\left(j_{k+2}-5 j_{k+1}\right) \\
& \times\left(\frac{1-j_{n+1}}{2 j_{n}-4}\right)^{k-1},
\end{aligned}
$$

while

$$
\operatorname{det} \Sigma=\operatorname{det} \Omega_{1}=(-1)^{((n-1)(n-2)) / 2} \text {, }
$$

and we have

$$
\begin{aligned}
\operatorname{det} B_{n}= & \left(1-j_{n+1}\right)^{n-1}+\left(2 j_{n}-4\right)^{n-2} \\
& \times \sum_{k=1}^{n-1}\left(j_{k+2}-5 j_{k+1}\right)\left(\frac{1-j_{n+1}}{2 j_{n}-4}\right)^{k-1} .
\end{aligned}
$$

Theorem 9. Let $B_{n}=\operatorname{Circ}\left(j_{1}, j_{2}, \ldots, j_{n}\right)$ be a circulant matrix; then $B_{n}$ is invertible for any positive integer $n$.

Proof. Since $j_{n}=\alpha^{n}+\beta^{n}$, where $\alpha+\beta=1, \alpha \beta=-2$. We have

$$
\begin{aligned}
& f\left(\omega^{k}\right)= \sum_{i=1}^{n} j_{i}\left(\omega^{k}\right)^{i-1}=\sum_{i=1}^{n}\left(\alpha^{i}+\beta^{i}\right)\left(\omega^{k}\right)^{i-1} \\
&= \frac{\alpha\left(1-\alpha^{n}\right)}{1-\alpha \omega^{k}}+\frac{\beta\left(1-\beta^{n}\right)}{1-\beta \omega^{k}} \\
&= \frac{(\alpha+\beta)-2 \alpha \beta \omega^{k}-\left(\alpha^{n+1}+\beta^{n+1}\right)}{1-(\alpha+\beta) \omega^{k}+\alpha \beta \omega^{2 k}} \\
&+\frac{\alpha \beta\left(\alpha^{n}+\beta^{n}\right) \omega^{k}}{1-(\alpha+\beta) \omega^{k}+\alpha \beta \omega^{2 k}} \\
&= \frac{1-j_{n+1}+\left(4-2 j_{n}\right) \omega^{k}}{1-\omega^{k}-2 \omega^{2 k}} \\
& k=1,2, \ldots, n-1 .
\end{aligned}
$$

If there exists $\omega^{l}(l=1,2, \ldots, n-1)$ such that $f\left(\omega^{l}\right)=0$, we obtain $1-j_{n+1}+\left(4-2 j_{n}\right) \omega^{l}=0$ for $1-\omega^{l}-2 \omega^{2 l} \neq 0$; thus, $\omega^{l}=\left(1-j_{n+1}\right) /\left(2 j_{n}-4\right)$ is a real number. While

$$
\omega^{l}=\exp \left(\frac{2 l \pi i}{n}\right)=\cos \frac{2 l \pi}{n}+i \sin \frac{2 l \pi}{n}
$$

$\sin (2 l \pi / n)=0$, so we have $\omega^{l}=-1$ for $0<2 l \pi / n<$ $2 \pi$. But $x=-1$ is not the root of the equation $1-j_{n+1}+$ $\left(4-2 j_{n}\right) x=0$ for any positive integer $n$. We obtain $f\left(\omega^{k}\right) \neq$ 0 for any $\omega^{k}(k=1,2, \ldots, n-1)$, while $f(1)=\sum_{i=1}^{n} j_{i}=$ $-(1 / 2)\left(5-j_{n+1}-2 j_{n}\right) \neq 0$. By Lemma 4 in [15], the proof is completed.

Lemma 10. Let the matrix $\Vdash=\left[h_{i j}\right]_{i, j=1}^{n-2}$ be of the form

$$
h_{i j}= \begin{cases}j_{1}-j_{n+1}, & i=j, \\ 4-2 j_{n}, & i=j+1, \\ 0, & \text { otherwise }\end{cases}
$$

and then the inverse $\mathbb{H}^{-1}=\left[h_{i j}^{\prime}\right]_{i, j=1}^{n-2}$ of the matrix $\mathbb{H}$ is equal to

$$
h_{i j}^{\prime}= \begin{cases}\frac{\left(2 j_{n}-4\right)^{i-j}}{\left(j_{1}-j_{n+1}\right)^{i-j+1}}, & i \geq j, \\ 0, & i<j .\end{cases}
$$

Proof. Let $r_{i j}=\sum_{k=1}^{n-2} h_{i k} h_{k j}^{\prime}$. Obviously, $r_{i j}=0$ for $i<j$. In the case $i=j$, we obtain

$$
r_{i i}=h_{i i} h_{i i}^{\prime}=\left(j_{1}-j_{n+1}\right) \cdot \frac{1}{j_{1}-j_{n+1}}=1 .
$$

For $i \geq j+1$, we obtain

$$
\begin{aligned}
r_{i j}= & \sum_{k=1}^{n-2} h_{i k} h_{k j}^{\prime}=h_{i, i-1} h_{i-1, j}^{\prime}+h_{i i} h_{i j}^{\prime} \\
= & \left(4-2 j_{n}\right) \cdot \frac{\left(2 j_{n}-4\right)^{i-j-1}}{\left(j_{1}-j_{n+1}\right)^{i-j}}+\left(j_{1}-j_{n+1}\right) \\
& \times \frac{\left(2 j_{n}-4\right)^{i-j}}{\left(j_{1}-j_{n+1}\right)^{i-j+1}}=0 .
\end{aligned}
$$

Hence, we verify $\mathbb{H} \mathbb{H}^{-1}=I_{n-2}$, where $I_{n-2}$ is $(n-2) \times(n-2)$ identity matrix. Similarly, we can verify $\mathbb{U}^{-1} \mathbb{H}=I_{n-2}$. Thus, the proof is completed.

Theorem 11. Let $B_{n}=\operatorname{Circ}\left(j_{1}, j_{2}, \ldots, j_{n}\right)$ be a circulant matrix; then we have

$$
B_{n}^{-1}=\frac{1}{\gamma_{n}} \operatorname{Circ}\left(y_{1}^{\prime}, y_{2}^{\prime}, \ldots, y_{n}^{\prime}\right)
$$


where

$$
\begin{aligned}
& y_{1}^{\prime}=1+\sum_{i=1}^{n-2} \frac{\left(j_{n+2-i}-5 j_{n+1-i}\right)\left(2 j_{n}-4\right)^{i-1}}{\left(j_{1}-j_{n+1}\right)^{i}}, \\
& y_{2}^{\prime}=-5+2 \sum_{i=1}^{n-2} \frac{\left(j_{n+1-i}-5 j_{n-i}\right)\left(2 j_{n}-4\right)^{i-1}}{\left(j_{1}-j_{n+1}\right)^{i}}, \\
& y_{k}^{\prime}=\frac{18\left(2 j_{n}-4\right)^{k-3}}{\left(j_{1}-j_{n+1}\right)^{k-2}}, \quad k=3,4, \ldots, n, \\
& \gamma_{n}=j_{1}-5 j_{n}+\sum_{k=1}^{n-2}\left(j_{k+2}-5 j_{k+1}\right)\left(\frac{2 j_{n}-4}{j_{1}-j_{n+1}}\right)^{n-(k+1)} .
\end{aligned}
$$

Proof. Let

$$
\Omega_{2}=\left(\begin{array}{ccccc}
1 & -\gamma_{n}^{\prime} & \omega_{13} & \cdots & \omega_{1 n} \\
0 & 1 & \omega_{23} & \cdots & \omega_{2 n} \\
0 & 0 & 1 & \cdots & 0 \\
0 & 0 & 0 & \cdots & 0 \\
\vdots & \vdots & \vdots & \ddots & \vdots \\
0 & 0 & 0 & \cdots & 1
\end{array}\right)
$$

where

$$
\begin{aligned}
& \omega_{1 j}=\frac{\gamma_{n}^{\prime}\left(j_{n+3-j}-5 j_{n+2-j}\right)}{\gamma_{n}}-j_{n+2-j}, \\
& \omega_{2 j}=-\frac{j_{n+3-j}-5 j_{n+2-j}}{\gamma_{n}}, \\
& \quad j=3,4, \ldots, n, \\
& \gamma_{n}=j_{1}-5 j_{n}+\sum_{k=1}^{n-2}\left(j_{k+2}-5 j_{k+1}\right)\left(\frac{2 j_{n}-4}{j_{1}-j_{n+1}}\right)^{n-(k+1)}, \\
& \gamma_{n}^{\prime}=\sum_{k=1}^{n-1} j_{k+1}\left(\frac{2 j_{n}-4}{j_{1}-j_{n+1}}\right)^{n-(k+1)} .
\end{aligned}
$$

We have

$$
\Sigma B_{n} \Omega_{1} \Omega_{2}=\mathscr{D}_{2} \oplus \mathbb{U},
$$

where $\mathscr{D}_{2}=\operatorname{diag}\left(j_{1}, \gamma_{n}\right)$ is a diagonal matrix and $\mathscr{D}_{2} \oplus \mathbb{H}$ is the direct sum of $\mathscr{D}_{2}$ and $\mathbb{H}$. If we denote $\Omega=\Omega_{1} \Omega_{2}$, then we obtain

$$
B_{n}^{-1}=\Omega\left(\mathscr{D}_{2}^{-1} \oplus \mathbb{\boxplus}^{-1}\right) \Sigma,
$$

since the last row entries of the matrix $\Omega$ are $0,1,\left(5 j_{n-1}-j_{n}\right) /$ $\gamma_{n},\left(5 j_{n-2}-j_{n-1}\right) / \gamma_{n}, \ldots,\left(5 j_{2}-j_{3}\right) / \gamma_{n}$. By Lemma 10 , if we let $B_{n}^{-1}=\operatorname{Circ}\left(y_{1}, y_{2}, \ldots, y_{n}\right)$, then its last row entries are given by the following equations:

$$
\begin{aligned}
y_{2}= & -\frac{5}{\gamma_{n}}+\frac{2}{\gamma_{n}} \sum_{i=1}^{n-2} \frac{\left(j_{n+1-i}-5 j_{n-i}\right)\left(2 j_{n}-4\right)^{i-1}}{\left(j_{1}-j_{n+1}\right)^{i}}, \\
y_{3}= & -\frac{j_{3}-5 j_{2}}{\gamma_{n}\left(j_{1}-j_{n+1}\right)}, \\
y_{4}= & \frac{j_{3}-5 j_{2}}{\gamma_{n}\left(j_{1}-j_{n+1}\right)} \\
& -\frac{1}{\gamma_{n}} \sum_{i=1}^{2} \frac{\left(j_{5-i}-5 j_{4-i}\right)\left(2 j_{n}-4\right)^{i-1}}{\left(j_{1}-j_{n+1}\right)^{i}}, \\
y_{5}= & \frac{2\left(j_{3}-5 j_{2}\right)}{\gamma_{n}\left(j_{1}-j_{n+1}\right)} \\
& +\frac{1}{\gamma_{n}} \sum_{i=1}^{2} \frac{\left(j_{5-i}-5 j_{4-i}\right)\left(2 j_{n}-4\right)^{i-1}}{\left(j_{1}-j_{n+1}\right)^{i}} \\
& -\frac{1}{\gamma_{n}} \sum_{i=1}^{3} \frac{\left(j_{6-i}-5 j_{5-i}\right)\left(2 j_{n}-4\right)^{i-1}}{\left(j_{1}-j_{n+1}\right)^{i}},
\end{aligned}
$$

$$
\begin{aligned}
y_{n}= & \frac{2}{\gamma_{n}} \sum_{i=1}^{n-4} \frac{\left(j_{n-1-i}-5 j_{n-2-i}\right)\left(2 j_{n}-4\right)^{i-1}}{\left(j_{1}-j_{n+1}\right)^{i}} \\
& +\frac{1}{\gamma_{n}} \sum_{i=1}^{n-3} \frac{\left(j_{n-i}-5 j_{n-1-i}\right)\left(2 j_{n}-4\right)^{i-1}}{\left(j_{1}-j_{n+1}\right)^{i}} \\
& -\frac{1}{\gamma_{n}} \sum_{i=1}^{n-2} \frac{\left(j_{n+1-i}-5 j_{n-i}\right)\left(2 j_{n}-4\right)^{i-1}}{\left(j_{1}-j_{n+1}\right)^{i}}, \\
y_{1}= & \frac{1}{\gamma_{n}}+\frac{2}{\gamma_{n}} \sum_{i=1}^{n-3} \frac{\left(j_{n-i}-5 j_{n-1-i}\right)\left(2 j_{n}-4\right)^{i-1}}{\left(j_{1}-j_{n+1}\right)^{i}} \\
& +\frac{1}{\gamma_{n}} \sum_{i=1}^{n-2} \frac{\left(j_{n+1-i}-5 j_{n-i}\right)\left(2 j_{n}-4\right)^{i-1}}{\left(j_{1}-j_{n+1}\right)^{i}} .
\end{aligned}
$$

Let

$$
\begin{array}{r}
D_{n}^{(k)}=\sum_{i=1}^{k} \frac{\left(j_{k+3-i}-5 j_{k+2-i}\right)\left(2 j_{n}-4\right)^{i-1}}{\left(j_{1}-j_{n+1}\right)^{i}}, \\
(j=1,2, \ldots, n-2)
\end{array}
$$

and we have

$$
\begin{aligned}
D_{n}^{(1)}- & D_{n}^{(2)} \\
& =\frac{j_{3}-5 j_{2}}{j_{1}-j_{n+1}}-\sum_{i=1}^{2} \frac{\left(j_{5-i}-5 j_{4-i}\right)\left(2 j_{n}-4\right)^{i-1}}{\left(j_{1}-j_{n+1}\right)^{i}}
\end{aligned}
$$




$$
\begin{aligned}
& =\frac{18\left(2 j_{n}-4\right)}{\left(j_{1}-j_{n+1}\right)^{2}} \\
& 2 D_{n}^{(n-3)}+D_{n}^{(n-2)} \\
& =2 \sum_{i=1}^{n-3} \frac{\left(j_{n-i}-5 j_{n-i-1}\right)\left(2 j_{n}-4\right)^{i-1}}{\left(j_{1}-j_{n+1}\right)^{i}} \\
& +\sum_{i=1}^{n-2} \frac{\left(j_{n+1-i}-5 j_{n-i}\right)\left(2 j_{n}-4\right)^{i-1}}{\left(j_{1}-j_{n+1}\right)^{i}} \\
& =2 \sum_{i=1}^{n-3} \frac{\left(j_{n-i}-5 j_{n-i-1}\right)\left(2 j_{n}-4\right)^{i-1}}{\left(j_{1}-j_{n+1}\right)^{i}} \\
& +\sum_{i=1}^{n-3} \frac{\left(j_{n+1-i}-5 j_{n-i}\right)\left(2 j_{n}-4\right)^{i-1}}{\left(j_{1}-j_{n+1}\right)^{i}} \\
& =\sum_{i=1}^{n-3} \frac{\left(j_{n+2}-i\right)-5 j_{n+1-i}\left(2 j_{n}-4\right)^{i-1}}{\left(j_{1}-j_{n+1}\right)^{i}} \\
& +\frac{\left(j_{3}-5 j_{2}\right)\left(2 j_{n}-4\right)^{n-3}}{\left(j_{1}-j_{n+1}\right)^{n-2}} \\
& =\sum_{i=1}^{n-2} \frac{\left(j_{n+2-i}-5 j_{n+1-i}\right)\left(2 j_{n}-4\right)^{i-1}}{\left(j_{1}-j_{n+1}\right)^{n-2}} \\
& 2 D_{n}^{(k)}+D_{n}^{(k+1)}-D_{n}^{(k+2)} \\
& =2 \sum_{i=1}^{k} \frac{\left(j_{k+3-i}-5 j_{k+2-i}\right)\left(2 j_{n}-4\right)^{i-1}}{\left(j_{1}-j_{n+1}\right)^{i}} \\
& +\sum_{i=1}^{k+1} \frac{\left(j_{k+4-i}-5 j_{k+3-i}\right)\left(2 j_{n}-4\right)^{i-1}}{\left(j_{1}-j_{n+1}\right)^{i}} \\
& -\sum_{i=1}^{k+2} \frac{\left(j_{k+5-i}-5 j_{k+4-i}\right)\left(2 j_{n}-4\right)^{i-1}}{\left(j_{1}-j_{n+1}\right)^{i}} \\
& =\sum_{i=1}^{k}\left[\frac{\left(2 j_{k+3-i}-10 j_{k+2-i}\right)\left(2 j_{n}-4\right)^{i-1}}{\left(j_{1}-j_{n+1}\right)^{i}}\right. \\
& +\frac{\left(j_{k+4-i}-5 j_{k+3-i}\right)\left(2 j_{n}-4\right)^{i-1}}{\left(j_{1}-j_{n+1}\right)^{i}} \\
& \left.-\frac{\left(j_{k+5-i}-5 j_{k+4-i}\right)\left(2 j_{n}-4\right)^{i-1}}{\left(j_{1}-j_{n+1}\right)^{i}}\right] \\
& +\frac{\left(j_{3}-5 j_{2}\right)\left(2 j_{n}-4\right)^{k}}{\left(j_{1}-j_{n+1}\right)^{k+1}} \\
& -\frac{\left(j_{4}-5 j_{3}\right)\left(2 j_{n}-4\right)^{k}}{\left(j_{1}-j_{n+1}\right)^{k+1}}
\end{aligned}
$$

We obtain

$$
\begin{aligned}
& y_{1}=\frac{1+2 D_{n}^{(n-3)}+D_{n}^{(n-2)}}{\gamma_{n}}, \\
& y_{2}=\frac{2 D_{n}^{(n-2)}-5}{\gamma_{n}}, \\
& y_{3}=-\frac{D_{n}^{(1)}}{\gamma_{n}}, \\
& y_{4}=-\frac{D_{n}^{(2)}-D_{n}^{(1)}}{\gamma_{n}}, \\
& y_{k}=-\frac{D_{n}^{(i-2)}-D_{n}^{(i-3)}-2 D_{n}^{(i-4)}}{\gamma_{n}}, k=5,6, \ldots, n, \\
& B_{n}^{-1}=\frac{1}{\gamma_{n}} \operatorname{Circ}\left(y_{1}^{\prime}, y_{2}^{\prime}, \ldots, y_{n}^{\prime}\right),
\end{aligned}
$$

where

$$
\begin{aligned}
y_{1}^{\prime}= & 1+\sum_{i=1}^{n-2} \frac{\left(j_{n+2-i}-5 j_{n+1-i}\right)\left(2 j_{n}-4\right)^{i-1}}{\left(j_{1}-j_{n+1}\right)^{i}}, \\
y_{2}^{\prime}= & -5+2 \sum_{i=1}^{n-2} \frac{\left(j_{n+1-i}-5 j_{n-i}\right)\left(2 j_{n}-4\right)^{i-1}}{\left(j_{1}-j_{n+1}\right)^{i}}, \\
y_{k}^{\prime}= & \frac{18\left(2 j_{n}-4\right)^{k-3}}{\left(j_{1}-j_{n+1}\right)^{k-2}, \quad k=3,4, \ldots, n,} \\
\gamma_{n}= & j_{1}-5 j_{n} \\
& +\sum_{k=1}^{n-2}\left(j_{k+2}-5 j_{k+1}\right)\left(\frac{2 j_{n}-4}{j_{1}-j_{n+1}}\right)^{n-(k+1)} .
\end{aligned}
$$

\section{Left Circulant Matrix with the Jacobsthal and Jacobsthal-Lucas Numbers}

In this section, let $A_{n}^{\prime}=\operatorname{LCirc}\left(J_{1}, J_{2}, \ldots, J_{n}\right)$ and $B_{n}^{\prime}=$ $\operatorname{LCirc}\left(j_{1}, j_{2}, \ldots, j_{n}\right)$ be two left circulant matrices. By using the obtained conclusions, we give the determinant for the matrices $A_{n}^{\prime}$ and $B_{n}^{\prime}$. Then, we prove that $A_{n}^{\prime}$ is an invertible matrix for $n>2$ and $B_{n}^{\prime}$ is an invertible matrix for any positive integer $n$. The inverses of the matrices $A_{n}^{\prime}$ and $B_{n}^{\prime}$ are also presented.

According to Lemma 5 in [15] and Theorems 4, 5, and 7, we can obtain the following theorems. 
Theorem 12. Let $A_{n}^{\prime}=\operatorname{LCirc}\left(J_{1}, J_{2}, \ldots, J_{n}\right)$ be a left circulant matrix; then one can prove that

$$
\begin{aligned}
\operatorname{det} A_{n}^{\prime}= & (-1)^{((n-1)(n-2)) / 2} \\
& \times\left[\left(1-J_{n+1}\right)^{n-1}+\left(2 J_{n}\right)^{n-2} \sum_{k=1}^{n-1} 2 J_{k}\left(\frac{1-J_{n+1}}{2 J_{n}}\right)^{k-1}\right] .
\end{aligned}
$$

Theorem 13. Let $A_{n}^{\prime}=\operatorname{LCirc}\left(J_{1}, J_{2}, \ldots, J_{n}\right)$ be a left circulant matrix; if $n>2$, then $A_{n}^{\prime}$ is an invertible matrix.

Theorem 14. Let $A_{n}^{\prime}=\operatorname{LCirc}\left(J_{1}, J_{2}, \ldots, J_{n}\right),(n>2)$ be a left circulant matrix; then one has

$$
A_{n}^{\prime-1}=\frac{1}{\delta_{n}} \operatorname{LCirc}\left(z_{1}, z_{2}, \ldots, z_{n}\right)
$$

where

$$
\begin{aligned}
& z_{1}=1+2 \sum_{i=1}^{n-2} \frac{J_{n-i}\left(2 J_{n}\right)^{i-1}}{\left(J_{1}-J_{n+1}\right)^{i}}, \\
& z_{k}=-\frac{2\left(2 J_{n}\right)^{n-1-k}}{\left(J_{1}-J_{n+1}\right)^{n-k}}, \quad k=2,3, \ldots, n-1, \\
& z_{n}=-1+4 \sum_{i=1}^{n-2} \frac{J_{n-1-i}\left(2 J_{n}\right)^{i-1}}{\left(J_{1}-J_{n+1}\right)^{i}}, \\
& \delta_{n}=J_{1}-J_{n}+\sum_{k=1}^{n-2} 2 J_{k}\left(\frac{2 J_{n}}{J_{1}-J_{n+1}}\right)^{n-(k+1)} .
\end{aligned}
$$

By Lemma 5 in [15] and Theorems 8, 9, and 11, the following conclusions can be obtained.

Theorem 15. Let $B_{n}^{\prime}=\operatorname{LCirc}\left(j_{1}, j_{2}, \ldots, j_{n}\right)$ be a left circulant matrix; then one has

$$
\begin{aligned}
\operatorname{det} B_{n}^{\prime}= & (-1)^{((n-1)(n-2)) / 2} \\
& \times\left[\left(1-j_{n+1}\right)^{n-1}+\left(2 j_{n}-4\right)^{n-2}\right. \\
& \left.\times \sum_{k=1}^{n-1}\left(j_{k+2}-5 j_{k+1}\right)\left(\frac{1-j_{n+1}}{2 j_{n}-4}\right)^{k-1}\right],
\end{aligned}
$$

where $j_{n}$ is the nth Jacobsthal-Lucas number.

Theorem 16. Let $B_{n}^{\prime}=\operatorname{LCirc}\left(j_{1}, j_{2}, \ldots, j_{n}\right)$ be a left circulant matrix; then $B_{n}^{\prime}$ is invertible for any positive integer $n$.

Theorem 17. Let $B_{n}^{\prime}=\operatorname{LCirc}\left(j_{1}, j_{2}, \ldots, j_{n}\right)$ be a left circulant matrix; then one has

$$
B_{n}^{\prime-1}=\frac{1}{\gamma_{n}} \operatorname{LCirc}\left(z_{1}^{\prime}, z_{2}^{\prime}, \ldots, z_{n}^{\prime}\right)
$$

where

$$
\begin{aligned}
z_{1}^{\prime}= & 1+\sum_{i=1}^{n-2} \frac{\left(j_{n+2-i}-5 j_{n+1-i}\right)\left(2 j_{n}-4\right)^{i-1}}{\left(j_{1}-j_{n+1}\right)^{i}}, \\
z_{k}^{\prime}= & \frac{18\left(2 j_{n}-4\right)^{n-1-k}}{\left(j_{1}-j_{n+1}\right)^{n-k}}, \quad k=2,3, \ldots, n-1, \\
z_{n}^{\prime}= & -5+2 \sum_{i=1}^{n-2} \frac{\left(j_{n+1-i}-5 j_{n-i}\right)\left(2 j_{n}-4\right)^{i-1}}{\left(j_{1}-j_{n+1}\right)^{i}}, \\
\gamma_{n}= & j_{1}-5 j_{n} \\
& +\sum_{k=1}^{n-2}\left(j_{k+2}-5 j_{k+1}\right)\left(\frac{2 j_{n}-4}{j_{1}-j_{n+1}}\right)^{n-(k+1)} .
\end{aligned}
$$

\section{5. g-Circulant Matrix with the Jacobsthal and Jacobsthal-Lucas Numbers}

In this section, let $A_{g, n}=g$-Circ $\left(J_{1}, J_{2}, \ldots, J_{n}\right)$ and $B_{g, n}=$ $g$-Circ $\left(j_{1}, j_{2}, \ldots, j_{n}\right)$ be two $g$-circulant matrices. By using the obtained conclusions, we give a determinant formula for the matrices $A_{g, n}$ and $B_{g, n}$. Then, we prove that $A_{g, n}$ is an invertible matrix for $n>2$ and $B_{g, n}$ is an invertible matrix for any positive integer $n$ if $(n, g)=1$. The inverses of the matrices $A_{g, n}$ and $B_{g, n}$ are also presented.

From Lemmas 6 and 7 in [15] and Theorems 4, 5, and 7, we deduce the following results.

Theorem 18. Let $A_{g, n}=g-\operatorname{Circ}\left(J_{1}, J_{2}, \ldots, J_{n}\right)$ be a $g$ circulant matrix. Then one has

$$
\begin{aligned}
\operatorname{det} A_{g, n}= & \operatorname{det} \mathbb{Q}_{g} \\
& \cdot\left[\left(1-J_{n+1}\right)^{n-1}+\left(2 J_{n}\right)^{n-2} \sum_{k=1}^{n-1} 2 J_{k}\left(\frac{1-J_{n+1}}{2 J_{n}}\right)^{k-1}\right],
\end{aligned}
$$

where $J_{n}$ is the nth Jacobsthal number.

Theorem 19. Let $A_{g, n}=g$-Circ $\left(J_{1}, J_{2}, \ldots, J_{n}\right)$ be a $g$-circulant matrix and $(n, g)=1$, when $n>2, A_{g, n}$ is an invertible matrix.

Theorem 20. Let $A_{g, n}=g-\operatorname{Circ}\left(J_{1}, J_{2}, \ldots, J_{n}\right)(n>2)$ be a $g$-circulant matrix and $(n, g)=1$; one has

$$
A_{g, n}^{-1}=\frac{1}{\delta_{n}} \operatorname{Circ}\left(\lambda_{1}, \lambda_{2}, \ldots, \lambda_{n}\right) \mathbb{Q}_{g}^{T},
$$


where

$$
\begin{aligned}
& \lambda_{1}=1+2 \sum_{i=1}^{n-2} \frac{J_{n-i}\left(2 J_{n}\right)^{i-1}}{\left(J_{1}-J_{n+1}\right)^{i}}, \\
& \lambda_{2}=-1+4 \sum_{i=1}^{n-2} \frac{J_{n-1-i}\left(2 J_{n}\right)^{i-1}}{\left(J_{1}-J_{n+1}\right)^{i}}, \\
& \lambda_{k}=-\frac{2\left(2 J_{n}\right)^{k-3}}{\left(J_{1}-J_{n+1}\right)^{k-2}}, \quad k=3,4, \ldots, n, \\
& \delta_{n}=J_{1}-J_{n}+\sum_{k=1}^{n-2} 2 J_{k}\left(\frac{2 J_{n}}{J_{1}-J_{n+1}}\right)^{n-(k+1)} .
\end{aligned}
$$

Taking Lemmas 6 and 7 in [15] and Theorems 8, 9, and 11 into account, we have the following theorems.

Theorem 21. Let $B_{g, n}=g$-Circ $\left(j_{1}, j_{2}, \ldots, j_{n}\right)$ be a g-circulant matrix; then one has

$$
\begin{aligned}
\operatorname{det} B_{g, n}= & \operatorname{det} \mathbb{Q}_{g} \\
& \cdot\left[\left(1-j_{n+1}\right)^{n-1}+\left(2 j_{n}-4\right)^{n-2}\right. \\
& \left.\times \sum_{k=1}^{n-1}\left(j_{k+2}-5 j_{k+1}\right)\left(\frac{1-j_{n+1}}{2 j_{n}-4}\right)^{k-1}\right],
\end{aligned}
$$

where $j_{n}$ is the nth Jacobsthal-Lucas number.

Theorem 22. Let $B_{g, n}=g$-Circ $\left(j_{1}, j_{2}, \ldots, j_{n}\right)$ be a g-circulant matrix and $(n, g)=1$; then $B_{g, n}$ is invertible for any positive integer $n$.

Theorem 23. Let $B_{g, n}=g$-Circ $\left(j_{1}, j_{2}, \ldots, j_{n}\right)$ be a $g$-circulant matrix and $(n, g)=1$; then one has

$$
B_{g, n}^{-1}=\frac{1}{\gamma_{n}} \operatorname{Circ}\left(\lambda_{1}^{\prime}, \lambda_{2}^{\prime}, \ldots, \lambda_{n}^{\prime}\right) \mathbb{Q}_{g}^{T},
$$

where

$$
\begin{aligned}
\lambda_{1}^{\prime}= & 1+\sum_{i=1}^{n-2} \frac{\left(j_{n+2-i}-5 j_{n+1-i}\right)\left(2 j_{n}-4\right)^{i-1}}{\left(j_{1}-j_{n+1}\right)^{i}}, \\
\lambda_{k}^{\prime}= & \frac{18\left(2 j_{n}-4\right)^{n-1-k}}{\left(j_{1}-j_{n+1}\right)^{n-k}}, \quad k=2,3, \ldots, n-1, \\
\lambda_{n}^{\prime}= & -5+2 \sum_{i=1}^{n-2} \frac{\left(j_{n+1-i}-5 j_{n-i}\right)\left(2 j_{n}-4\right)^{i-1}}{\left(j_{1}-j_{n+1}\right)^{i}}, \\
\gamma_{n}= & j_{1}-5 j_{n} \quad \\
& +\sum_{k=1}^{n-2}\left(j_{k+2}-5 j_{k+1}\right)\left(\frac{2 j_{n}-4}{j_{1}-j_{n+1}}\right)^{n-(k+1)} .
\end{aligned}
$$

\section{Conclusion}

This paper obtains better results for the determinants and inverses of circulant type matrices by some properties of Jacobsthal and Jacobsthal-Lucas numbers. The reason we focus our attentions on circulant type matrices is to explore the application of circulant type matrices in solving fractional order differential equations.

\section{Conflict of Interests}

The authors declare that there is no conflict of interests regarding the publication of this paper.

\section{Acknowledgments}

The research is supported by the Development Project of Science \& Technology of Shandong Province (Grant no. 2012GGX10115) and NSFC (Grant no. 11301252) and the AMEP of Linyi University, China.

\section{References}

[1] W. Qu, S.-L. Lei, and S.-W. Vong, "Circulant and skew-circulant splitting iteration for fractional advection-diffusion equations," International Journal of Computer Mathematics, vol. 91, no. 10, pp. 2232-2242, 2014.

[2] E. Ahmed, A. M. El-Sayed, and H. A. El-Saka, "On some RouthHurwitz conditions for fractional order differential equations and their applications in Lorenz, Rössler, Chua and CHEn systems," Physics Letters A, vol. 358, no. 1, pp. 1-4, 2006.

[3] E. Ahmed and A. S. Elgazzar, "On fractional order differential equations model for nonlocal epidemics," Physica A: Statistical Mechanics and Its Applications, vol. 379, no. 2, pp. 607-614, 2007.

[4] S.-L. Lei and H.-W. Sun, "A circulant preconditioner for fractional diffusion equations," Journal of Computational Physics, vol. 242, pp. 715-725, 2013.

[5] P. E. Kloeden, A. Neuenkirch, and R. Pavani, "Multilevel Monte Carlo for stochastic differential equations with additive fractional noise," Annals of Operations Research, vol. 189, pp. 255-276, 2011.

[6] P. J. Davis, Circulant Matrices, John Wiley \& Sons, New York, NY, USA, 1979.

[7] Z. L. Jiang and Z. X. Zhou, Circulant Matrices, Chengdu Technology University Publishing Company, Chengdu, China, 1999.

[8] C. Erbas and M. M. Tanik, "Generating solutions to the Nqueens problem using 2-circulants," Mathematics Magazine, vol. 68, no. 5, pp. 343-356, 1995.

[9] Y. K. Wu, R. Z. Jia, and Q. Li, " $g$-circulant solutions to the $(0 ; 1)$ matrix equation $A^{m}=J_{n}^{*}$, Linear Algebra and Its Applications, vol. 345, pp. 195-224, 2002.

[10] E. Ngondiep, S. Serra-Capizzano, and D. Sesana, "Spectral features and asymptotic properties for $g$-circulants and $g$ Toeplitz sequences," SIAM Journal on Matrix Analysis and Applications, vol. 31, no. 4, pp. 1663-1687, 2010.

[11] A. Bose, R. S. Hazra, and K. Saha, "Spectral norm of circulanttype matrices," Journal of Theoretical Probability, vol. 24, no. 2, pp. 479-516, 2011. 
[12] A. F. Horadam, "Jacobsthal representation numbers," The Fibonacci Quarterly. The Official Journal of the Fibonacci Association, vol. 34, no. 1, pp. 40-54, 1996.

[13] A. F. Horadam, "Jacobsthal and Pell curves," The Fibonacci Quarterly, vol. 26, no. 1, pp. 79-83, 1988.

[14] Z. L. Jiang, J. J. Yao, and F. L. Lu, "On skew circulant type matrices involving any continuous Fibonacci numbers," Abstract and Applied Analysis, vol. 2014, Article ID 483021, 10 pages, 2014.

[15] Z. Jiang, Y. Gong, and Y. Gao, "Circulant type matrices with the sum and product of Fibonacci and Lucas numbers," Abstract and Applied Analysis, vol. 2014, Article ID 375251, 12 pages, 2014.

[16] X. Jiang and K. Hong, "Exact determinants of some special circulant matrices involving four kinds of famous numbers," Abstract and Applied Analysis, vol. 2014, Article ID 273680, 12 pages, 2014.

[17] D. V. Jaiswal, "On determinants involving generalized Fibonacci numbers," The Fibonacci Quarterly, vol. 7, pp. 319-330, 1969.

[18] D. A. Lind, "A Fibonacci circulant," The Fibonacci Quarterly, vol. 8, no. 5, pp. 449-455, 1970.

[19] L. Dazheng, "Fibonacci-Lucas quasi-cyclic matrices," The Fibonacci Quarterly, vol. 40, no. 3, pp. 280-286, 2002.

[20] S.-Q. Shen, J.-M. Cen, and Y. Hao, "On the determinants and inverses of circulant matrices with Fibonacci and Lucas numbers," Applied Mathematics and Computation, vol. 217, no. 23, pp. 9790-9797, 2011.

[21] H. Karner, J. Schneid, and C. W. Ueberhuber, "Spectral decomposition of real circulant matrices," Linear Algebra and its Applications, vol. 367, pp. 301-311, 2003.

[22] W. T. Stallings and T. L. Boullion, "The pseudoinverse of an $r$-circulant matrix," Proceedings of the American Mathematical Society, vol. 34, pp. 385-388, 1972. 


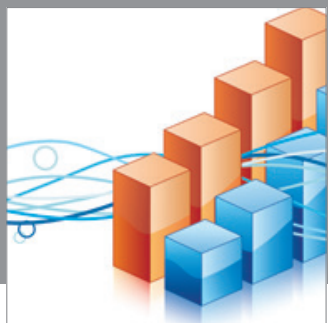

Advances in

Operations Research

mansans

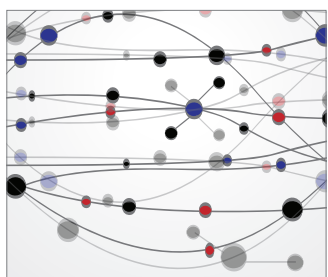

The Scientific World Journal
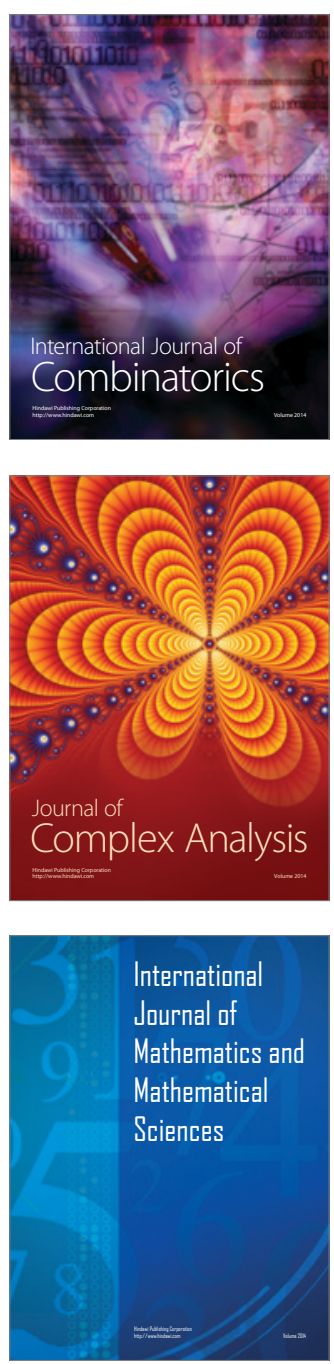
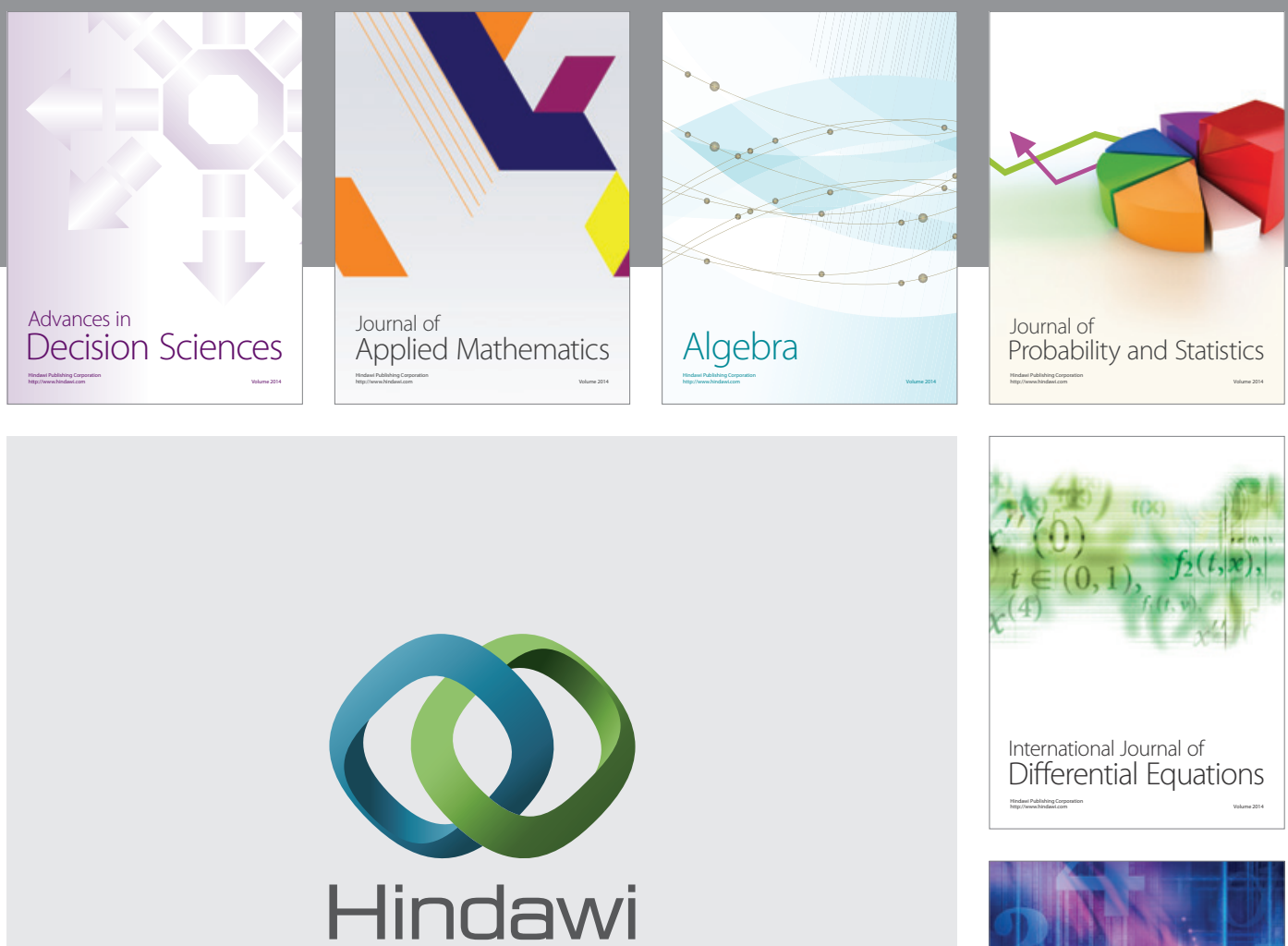

Submit your manuscripts at http://www.hindawi.com
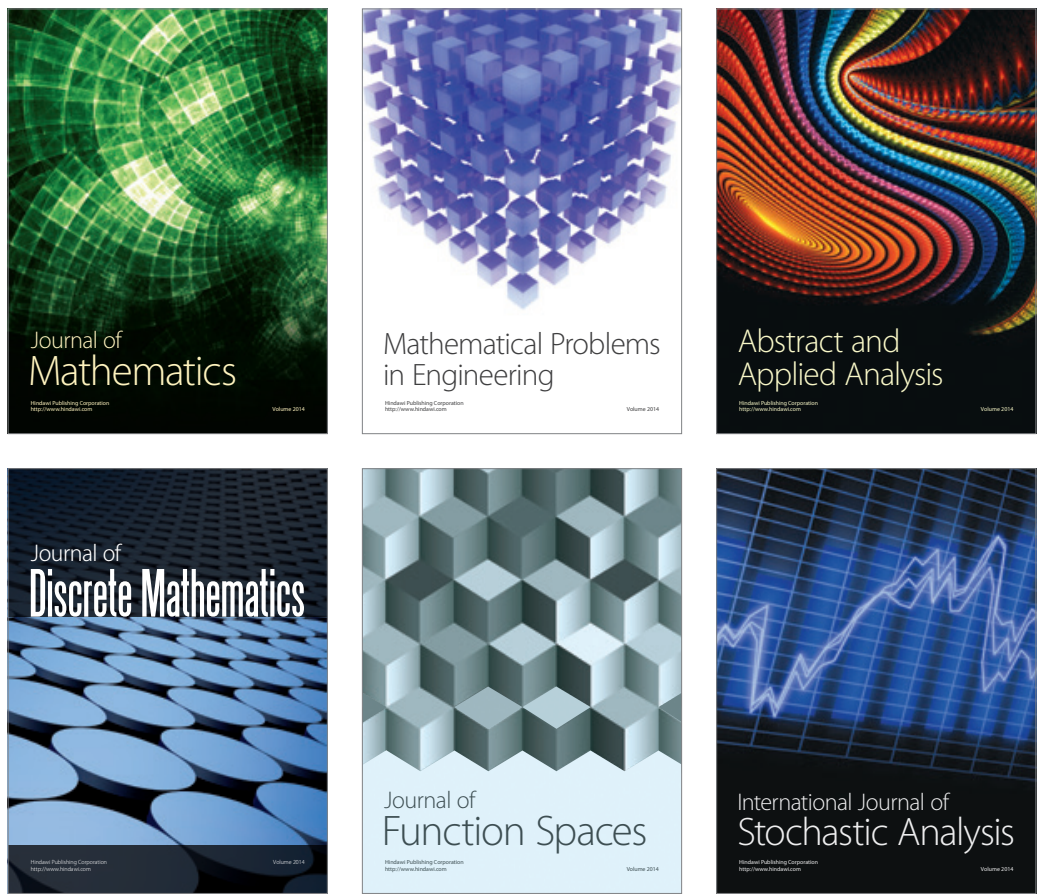

Journal of

Function Spaces

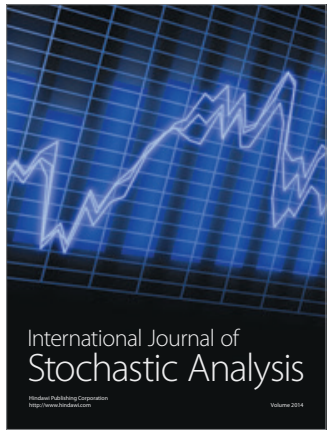

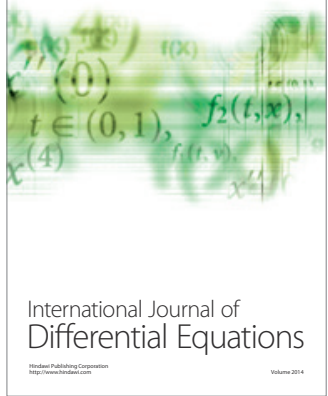
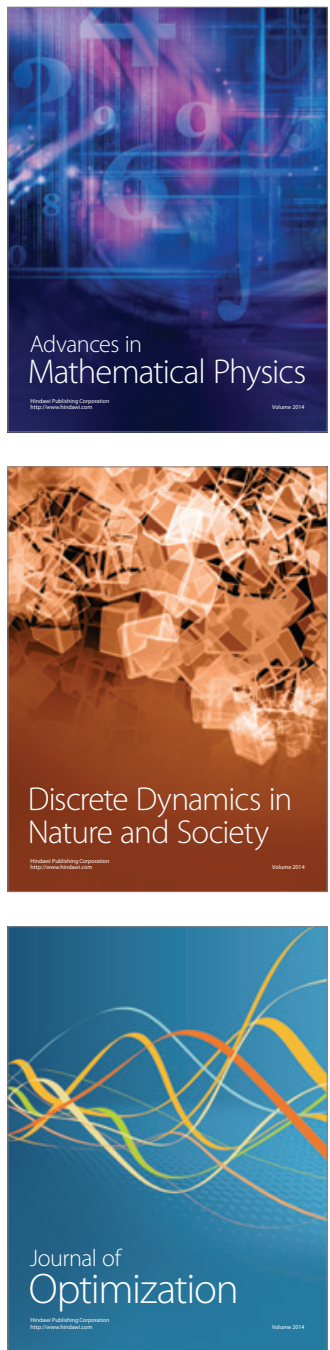